\title{
Novel Mott Transitions in a Nonisotropic Two-Band Hubbard Model
}

\author{
A. Liebsch* \\ Institut für Festkörperforschung, Forschungszentrum Jülich, 52425 Jülich, Germany
}

(Received 23 May 2005; published 8 September 2005)

\begin{abstract}
The Mott transition in a two-band Hubbard model involving subbands of different widths is studied as a function of temperature using dynamical mean field theory combined with exact diagonalization. The phase diagram is shown to exhibit two successive first-order transitions if the full Hund's rule coupling is included. In the absence of spin-flip and pair-exchange terms the lower transition remains first-order while the upper becomes continuous.
\end{abstract}

DOI: 10.1103/PhysRevLett.95.116402

PACS numbers: 71.20.Be, 71.27.+a

The nature of the metal-insulator transition in materials involving subbands of different widths has been intensively debated during recent years [1-12]. This issue is relevant for the understanding of the effect of strong local Coulomb interactions in systems such as $\mathrm{Ca}_{2-x} \mathrm{Sr}_{x} \mathrm{RuO}_{4}$. In this perovskite layer the partially filled Ru $t_{2 g}$ bands for $x=2$ consist of wide $d_{x y}$ and narrow $d_{x z, y z}$ bands [13]. As pointed out in [14], the onsite Coulomb energy lies between the subband widths: $W_{x z, y z}<U<W_{x y}$. The usual criterion with the parameter $U / W$ as a measure of the importance of correlations must then be generalized. The pure $\mathrm{Sr}$ compound is superconducting below $1.5 \mathrm{~K}$ [15]. Isoelectronic replacement of $\mathrm{Sr}$ by $\mathrm{Ca}$ leads to an effective band narrowing due to octahedral distortions [16] and a metal-insulator transition [17]. As a consequence of noncubic crystal fields many other transition metal oxides also involve nonequivalent partially occupied subbands.

A key question in these materials is whether the wide and narrow subbands exhibit separate Mott transitions or whether single-particle hybridization and interorbital Coulomb interactions ensure a single transition for all bands simultaneously. This issue was studied initially by Anisimov et al. [1] and Liebsch [2] for $\mathrm{Ca}_{2-x} \mathrm{Sr}_{x} \mathrm{RuO}_{4}$ using simplified band structure models which did not yet include the full complexity due to Ca-induced octahedral distortions. Correlations were treated in the dynamical mean field theory [18] (DMFT) combined with the noncrossing approximation (NCA) and quantum Monte Carlo (QMC) method, respectively. Since the NCA calculations (at $T=0$ ) neglected interorbital Coulomb interactions the results showed separate, "orbital-selective" Mott transitions for the narrow and wide subbands [1]. In contrast, the QMC calculations (at $T=0.125 \mathrm{eV}$ ) included interorbital Coulomb interactions and suggested a common transition for all $t_{2 g}$ bands [2].

Recent theoretical studies of the Mott transition in a paramagnetic two-band model system have led to apparent contradictions [3-11]. Including the full Hund's rule coupling Koga et al. [4] found orbital-selective metal-insulator transitions at $T=0$. Neglecting spin-flip and pairexchange terms Liebsch [5] obtained at $T>0$ a single first-order transition, followed by a bad-metallic-nonFermi-liquid (NFL) phase. As we argue below, the various results and conclusions are consistent provided that the choice of crucial parameters such as temperature and Hund's rule coupling is properly taken into account.

In the present work we combine finite temperature DMFT with exact diagonalization [19] (ED) to determine the $T / U$ phase diagram of a two-band system consisting of nonhybridizing, half-filled subbands with semicircular density of states of width $W_{1}=2 \mathrm{eV}$ and $W_{2}=4 \mathrm{eV}$. The subbands interact via intraorbital and interorbital Coulomb matrix elements $U$ and $U^{\prime}=U-2 J$, where $J$ is the Hund's rule exchange integral. In contrast to the multiband QMC approach, which includes only Ising-like exchange terms to avoid sign problems at low temperatures [20], ED permits also the consideration of spin-flip and pair-exchange interactions. To be specific we take $J=$ $U / 4$ which is approximately satisfied in several transition metal oxides.

The main result of this work is that the $T / U$ phase diagram in the presence of the full Hund's rule exchange exhibits two successive first-order phase transitions, with separate hysteresis loops and coexistence regions. The intermediate region corresponds to the $T>0$ analog of the orbital-selective Mott (OSM) phase obtained at $T=0$ in [4]. On the other hand, if spin-flip and pair-exchange terms are omitted, we find a single first-order transition succeeded by a non-Fermi-liquid phase, in agreement with previous QMC results [5]. Both trends are consistent with those obtained by several groups $[4,6,8-10]$ for the same two-band model at $T=0$.

The ED/DMFT results are derived from a two-band generalization of the approach employed for single bands $[18,19,21]$. Since at $T>0$ all states of the impurity Hamiltonian are used in the construction of the subband Green's functions $G_{i}\left(i \omega_{n}\right)$, two bath levels per impurity level are taken into account $\left(n_{s}=6\right.$ per spin). To check the accuracy of this approximation we have evaluated the $T / U$ phase diagram of a single band for $n_{s}=3, \ldots, 6$. As shown in Fig. 1, the stability boundaries $U_{c 1}(T)$ and $U_{c 2}(T)$ for $n_{s}=3$ are slightly too low. Nevertheless, the overall shape 


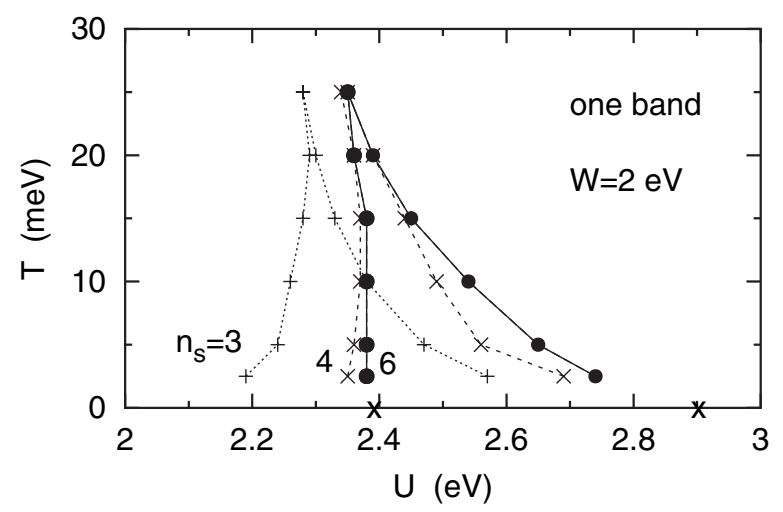

FIG. 1. Phase diagram for a one-band Hubbard model, calculated within ED/DMFT for $n_{s}=3,4,6$. symbols $\times$ at $T=0$ : results obtained in [35].

of the phase diagram agrees qualitatively with the converged results for $n_{s}=6$ [22]. Thus, we are confident that in the two-band case $n_{s}=6$ also yields a reasonable picture of the phase diagram. Preliminary results for $n_{s}=$ 8 will also be presented.

To analyze the metal-insulator transition we study the quasiparticle weights $Z_{i}=1 /\left[1-d \operatorname{Re} \Sigma_{i}(\omega) /\left.d \omega\right|_{\omega=0}\right]$, which in the metallic range can be represented as $Z_{i} \approx$ $1 /\left[1-\operatorname{Im} \Sigma_{i}\left(i \omega_{0}\right) / \omega_{0}\right]$, where $\Sigma_{i}\left(i \omega_{0}\right)$ is the subband self-energy at the first Matsubara frequency. Figure 2(a) shows $Z_{i}(U)$ for $J^{\prime}=J=U / 4$, where $J^{\prime}$ denotes spin-flip and pair-exchange terms [23]. Two critical regions can be identified, each with hysteresis loops characteristic of firstorder phase transitions. The coexistence areas are $U_{c 1}^{<}(T)<U<U_{c 2}^{<}(T)$ near $2.0 \mathrm{eV}$ and $U_{c 1}^{>}(T)<U<$ $U_{c 2}^{>}(T)$ near $3.0 \mathrm{eV}$, where $U_{c n}^{<}(T)$ and $U_{c n}^{>}(T)$ are the stability boundaries obtained for increasing $(n=2)$ and decreasing $(n=1) U$. Let us denote the true critical energies of these transitions as $U_{c}^{<}(T)$ and $U_{c}^{>}(T)$. Below

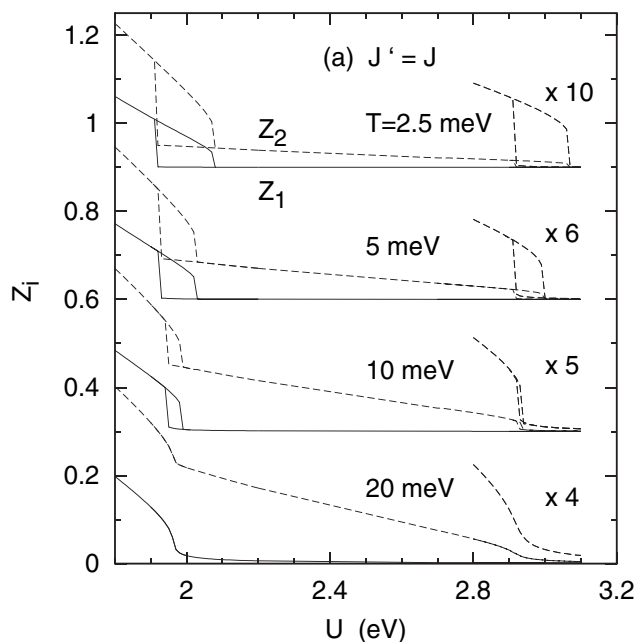

$U_{c}^{<}(T)$ both bands are metallic while above $U_{c}^{>}(T)$ both are insulating. At the lower transition both bands undergo firstorder transitions - but in fundamentally different ways: $Z_{1}(U)$ becomes very small while $Z_{2}(U)$ drops to a finite value. The narrow band therefore undergoes a "complete" metal-insulator transition, whereas the wide band exhibits an "incomplete" transition to a new, considerably more correlated phase. This band becomes fully insulating near $3.0 \mathrm{eV}$, where it exhibits a weak second hysteresis loop. To our knowledge this is the first time that sequential firstorder transitions are identified in the $T / U$ phase diagram of a Hubbard model involving nonequivalent bands [24]. If such a material could be encountered experimentally, the conductivity as a function of pressure would show two consecutive jumps.

Figure 2(b) shows $Z_{i}(U)$ for $J^{\prime}=0, J=U / 4$, i.e., in the absence of spin-flip and pair-exchange terms. The results are similar to those in Fig. 2(a), with the important exception that the wide band above the lower transition is even more correlated and the upper transition is now continuous at smaller $U$ [25]. The lower transition remains first-order for both subbands. In this case the conductivity shows a jump at $U_{c}^{<}(T)$ but a change of slope at $U_{c}^{>}(T)$. The results in Fig. 2(b) confirm the picture obtained previously within the QMC for $T>0, J^{\prime}=0$ [5,26], showing a single first-order transition near $U=2.1 \mathrm{eV}$ followed by a mixed insulating/bad-metallic phase which becomes fully insulating at $U=2.7 \mathrm{eV}$ [27]. Figure 2(b) also demonstrates that the two-band ED/DMFT for $n_{s}=6$ is qualitatively accurate [28].

The phase diagrams deduced from the ED results for $T \geq 2.5 \mathrm{meV}$ are shown in Fig. 3. For $J^{\prime}=J$ as well as $J^{\prime}=0$ the transition at $U_{c}^{<}(T)$ is first order for both subbands. The subsequent transition of the wide band at $U_{c}^{>}(T)$ is first order for $J^{\prime}=J$ but continuous for $J^{\prime}=0$. At $U_{c}^{<}(T)$ the metal-insulator transition is complete only

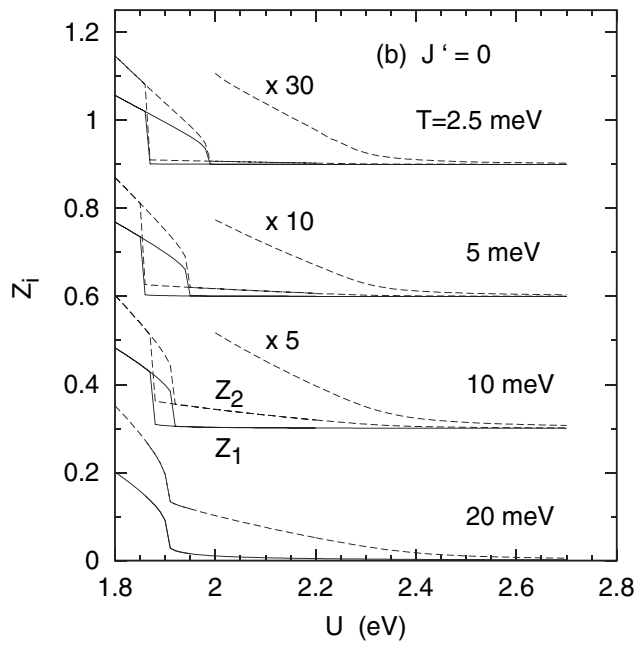

FIG. 2. $Z_{i}(U)$ of nonisotropic two-band Hubbard model, calculated within ED/DMFT. (a) $J^{\prime}=J=U / 4$, (b) $J^{\prime}=0, J=U / 4$. Solid (dashed) curves: narrow (wide) band. Results for different $T$ are displaced vertically by 0.3 . 

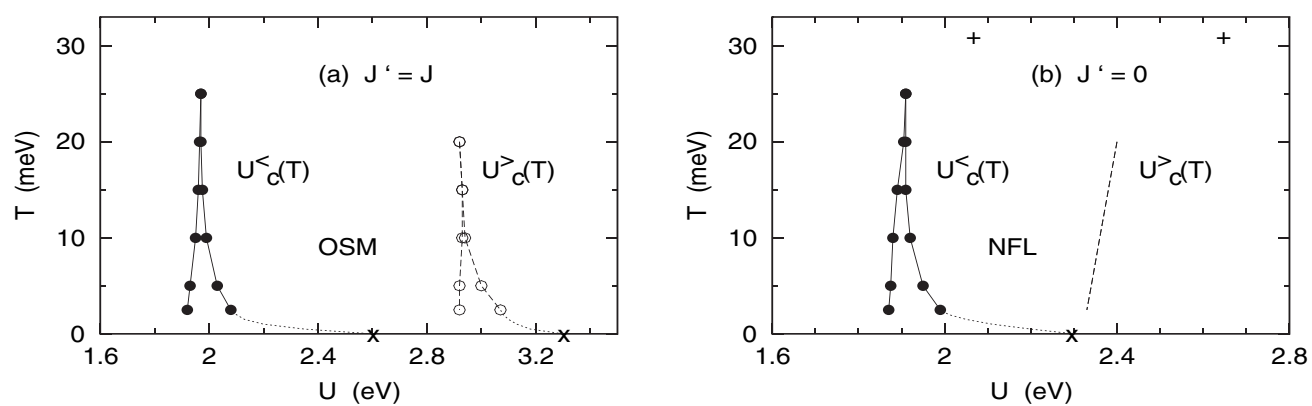

FIG. 3. Phase diagram for nonisotropic two-band Hubbard model, calculated within ED/DMFT. (a) $J^{\prime}=J=U / 4$, (b) $J^{\prime}=0, J=$ $U / 4$. Solid dots in (a), (b) stability boundaries of both subbands near lower first-order transition. Open dots in (a): stability boundaries of wide band near the upper first-order transition. Dashed line in (b): approximate location of continuous transition of wide band. symbols $(\times): T=0$ transitions obtained in [4,6,10]; (+): results for $T=31 \mathrm{meV}$ obtained in [5,11]. Lines are guides to the eye.

for the narrow band. The wide band first undergoes a transition to a more strongly correlated phase and becomes truly insulating at the second transition at $U_{c}^{>}(T)$. The overall shape of the phase diagram for $J^{\prime}=J$ agrees with the one recently obtained by Inaba et al. [24].

Figure 3 also shows the critical Coulomb energies obtained at $T=0[4,6,10]$ and $T=31 \mathrm{meV}[5,11]$. As illustrated in Fig. 1 for the one-band case, the ED scheme with small $n_{s}$ underestimates the critical Coulomb energies by about 0.1 to $0.2 \mathrm{eV}$. Preliminary results for $n_{s}=8$ indicate similar shifts in the two-band case. Taking these displacements into account the ED results shown in Fig. 3 are in excellent correspondence with those obtained at $T=0$ and $T=31 \mathrm{meV}$. Evidently the conflicting conclusions reached in [1-6,8-11] concerning the nature of the Mott transition in multiband systems were caused by different behaviors obtained for $T=0$ vs $T>0$ and $J^{\prime}=J$ vs $J^{\prime}=$ 0 . Accounting for these different parameter choices, the DMFT treatments are consistent.

Quasiparticle spectra derived within the QMC/DMFT for $J^{\prime}=0$ [5] showed that in the intermediate phase the self-energy of the wide band at small $\omega_{n}$ deviates significantly from metallic $\sim \omega_{n}$ behavior [29]. Accordingly, the quasiparticle spectra show a pseudogap which for larger $U$ gets more pronounced [30], indicating progressive badmetal-non-Fermi-liquid properties. A true gap opens at $U_{c}^{>}(T)$. Thus, $Z_{2}(U)>0$ in the region $U_{c}^{<}(T)<U<$ $U_{c}^{>}(T)$ does not imply existence of quasiparticles. As indicated in Fig. 3(b), in the absence of spin flip and pair exchange the intermediate phase is a mixed insulating/NFL phase and not an OSM phase with coexisting insulating and FL-metallic subbands. The same trend is found using the $T>0$ ED/DMFT [31].

For $J^{\prime}=J$, the low-frequency analysis of $\Sigma_{2}\left(i \omega_{n}\right)$ is more intricate. As shown in Fig. 4(a) for $n_{s}=6, \Sigma_{2}\left(i \omega_{n}\right)$ reveals deviations from metallic $\sim \omega_{n}$ variation, giving rise to small pseudogaps in the quasiparticle spectra. This behavior is incompatible with $\operatorname{Im} \Sigma_{2}(\omega) \sim \omega^{2}$ and suggests that, as for $J^{\prime}=0$, in the intermediate phase the wide band at $T>0$ violates Fermi-liquid theory. The extension of the present ED approach to $n_{s}=8$ indicates, however, that the additional bath levels are important for the low- $\omega_{n}$ variation of $\Sigma_{2}\left(i \omega_{n}\right)$. As shown in Fig. 4(b), the deviations are absent and the $\sim \omega_{n}$ variation is consistent with metallic behavior. In fact, the shoulder near $\omega_{0}=0.06$ suggests that Fermi-liquid properties persist to about $T=\omega_{0} / \pi \approx$ $20 \mathrm{meV}$. Thus, the OSM phase in Fig. 3(a) is the $T>0$ analog of the orbital-selective Mott phase identified first by Koga et al. [4] at $T=0$. A more complete discussion of the results for $n_{s}=8$ will be given elsewhere [31]. Because of finite size limitations of the present ED/DMFT scheme associated with the small number of bath levels, a precise determination of low-temperature properties is not possible. Nevertheless, an approximate extrapolation of $\Sigma_{2}\left(i \omega_{n}\right)$ indicates that the $T \rightarrow 0$ limit for $J^{\prime}=J$ with $Z_{2} \approx 0.1$ agrees with previous $T=0$ studies [4,6,8-10].

In view of the importance of spin-flip and pair-exchange terms for the Mott transition in multiband materials [32] it is desirable to investigate the $T / U$ phase diagram by methods which permit adequate treatment of the complete Hund's rule matrix, for instance, a two-band extension of $T>0$ numerical renormalization group studies [33]; see also [24]. Because of sign problems, recent QMC exten-
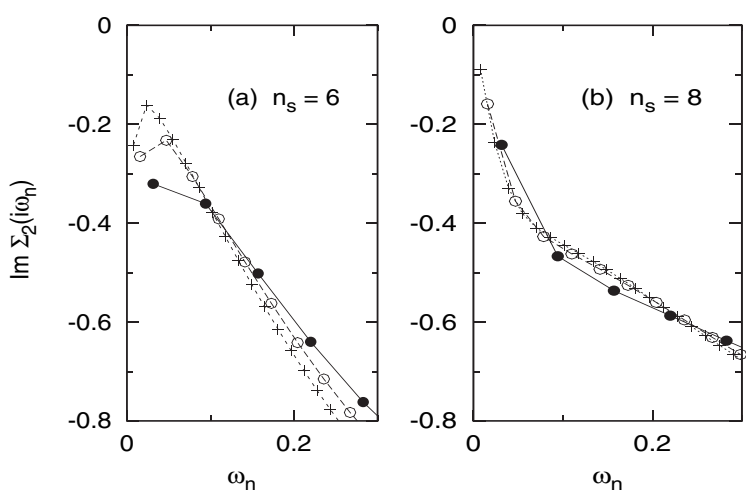

FIG. 4. Self-energy of wide band for $J^{\prime}=J$ in intermediate phase at $U=2.4 \mathrm{eV}$. (a) $n_{s}=6$; (b) $n_{s}=8$. Solid dots: $T=10 \mathrm{meV}$; empty dots: $T=5 \mathrm{meV}$; $+: T=2.5 \mathrm{meV}$. 
sions including spin-flip and pair-exchange terms are limited to $T \geq 1 / 6 \mathrm{eV} \gg T_{c}$ [7] or $T=0$ [10]. Also, other recent works $[8,9]$ employing a variety of quantum impurity methods deal so far mainly with $T=0$ and do not yet allow the identification of the multiband Mott transition at general $T, U$ values.

In summary, the $T / U$ phase diagram of the Hubbard model involving half-filled, nonequivalent subbands is shown to be remarkably rich. The competing kinetic energy scales, coupled via Coulomb and exchange energies, give rise to sequential first-order phase transitions. The lower transition separates a purely metallic phase from a phase with insulating narrow and strongly correlated, metallic wide subbands. The wide band becomes insulating at the second first-order transition. Omission of spin-flip and pair-exchange terms enhances the correlations in the wide band in the intermediate phase so that it no longer satisfies Fermi-liquid criteria, and modifies the upper phase transition from first-order to continuous.

For the analysis of experimental data of materials such as $\mathrm{Ca}_{2-x} \mathrm{Sr}_{x} \mathrm{RuO}_{4}$ it is necessary to account also for hybridization between orbitals. Preliminary studies of this effect within two-band models for $T \gg T_{c}$ [7] and $T=0$ $[9,12]$ suggest significant changes. Moreover, spatial fluctuations [34] and deviations from half-filling might play a decisive role close to the Mott transition. More work is needed to investigate whether both first-order transitions persist in the presence of these effects, or whether the weak first-order behavior of the upper transition disappears and only the dominant lower transition survives as the common first-order Mott transition for all bands.

I would like to thank T. Costi, K. Held, N. Kawakami, and E. Koch for valuable discussions.

*Email address: a.liebsch@fz-juelich.de

[1] V.I. Anisimov, I. A. Nekrasov, D. E. Kondakov, T. M. Rice, and M. Sigrist, Eur. Phys. J. B 25, 191 (2002).

[2] A. Liebsch, Europhys. Lett. 63, 97 (2003).

[3] A. Liebsch, Phys. Rev. Lett. 91, 226401 (2003).

[4] A. Koga, N. Kawakami, T. M. Rice, and M. Sigrist, Phys. Rev. Lett. 92, 216402 (2004).

[5] A. Liebsch, Phys. Rev. B 70, 165103 (2004).

[6] A. Koga, N. Kawakami, T. M. Rice, and M. Sigrist, condmat/0406457.

[7] A. Koga, N. Kawakami, T. M. Rice, and M. Sigrist, Phys. Rev. B 72, 045128 (2005).

[8] M. Ferrero, F. Becca, M. Fabrizio, and M. Capone, condmat/0503759 [Phys. Rev. B (to be published)].

[9] L. de' Medici, A. Georges, and S. Biermann, cond-mat/ 0503764 [Phys. Rev. B (to be published)].

[10] R. Arita and K. Held, cond-mat/0504040.

[11] C. Knecht, N. Blümer, and P. G. J. van Dongen, cond-mat/ 0505106 [Phys. Rev. B (to be published)].
[12] Yun Song and L.-J. Zou, Phys. Rev. B 72, 085114 (2005).

[13] T. Oguchi, Phys. Rev. B 51, 1385 (1995); I. I. Mazin and D. Singh, Phys. Rev. Lett. 79, 733 (1997).

[14] A. Liebsch and A. I. Lichtenstein, Phys. Rev. Lett. 84, 1591 (2000).

[15] Y. Maeno, T. M. Rice, and M. Sigrist, Phys. Today 54, No. 1, 42 (2001).

[16] Z. Fang and K. Terakura, Phys. Rev. B 64, R020509 (2001); Z. Fang, N. Nagaosa, and K. Terakura, Phys. Rev. B 69, 045116 (2004).

[17] S. Nakatsuji and Y. Maeno, Phys. Rev. Lett. 84, 2666 (2000).

[18] For a review, see A. Georges, G. Kotliar, W. Krauth, and M. J. Rozenberg, Rev. Mod. Phys. 68, 13 (1996).

[19] M. Caffarel and W. Krauth, Phys. Rev. Lett. 72, 1545 (1994).

[20] For a discussion of this problem, see K. Held and D. Vollhardt, Eur. Phys. J. B 5, 473 (1998).

[21] I thank A. Georges, G. Kotliar, W. Krauth, and M.J. Rozenberg for making the single-band ED computer code available, and A.I. Lichtenstein for parts of the two-band ED computer code.

[22] N. H. Tong, S. Q. Shen, and F. C. Pu, Phys. Rev. B 64, 235109 (2001).

[23] More accurate results at low $\omega_{n}$ and $T$ are obtained by minimizing differences between impurity and Anderson model Green's functions via $\sum_{n}\left|\Delta G_{i}\left(i \omega_{n}\right)\right|^{2}$ rather than $\sum_{n}\left|\Delta G_{i}\left(i \omega_{n}\right)\right|$. E. Koch (private communication).

[24] After completion of this work we learned of similar results by K. Inaba et al., cond-mat/0506151.

[25] The term "continuous" indicates here that there is no jump; it may also signify crossover behavior.

[26] Surprisingly, two-band DMFT using iterated perturbation theory (IPT) also reveals a similar picture for $J^{\prime}=J$ [5].

[27] The QMC results in [5] are in good agreement with those of [11]. The comparison of $Z_{i}(U)$ and $N_{i}(\omega)$ in Fig. (9b) in Ref. [5] with Figs. 1(b)(inset), 3, and 4 in Ref. [11] is provided in A. Liebsch, cond-mat/0506138; Thus, the claims in [11] that "the second transition was not seen in earlier QMC and IPT studies" and "our high-precision data correct earlier QMC results" are unfounded.

[28] The QMC hysteresis region is slightly wider than in ED. This could be due to slow convergence of QMC at $T=$ $0.02 \mathrm{eV}$ and too small $n_{s}$ in ED.

[29] Similar QMC results for $J^{\prime}=0, T>0, W_{1}=0.1 W_{2}$ were shown to be closely related to the ferromagnetic Kondo lattice. See S. Biermann et al., cond-mat/0505737.

[30] See also Fig. 3 of Ref. [11].

[31] A. Liebsch (to be published).

[32] See also Th. Pruschke and R. Bulla, Eur. Phys. J. B 44, 217 (2005).

[33] T. A. Costi (to be published).

[34] O. Parcollet, G. Biroli, and G. Kotliar, Phys. Rev. Lett. 92, 226402 (2004).

[35] See R. Bulla, T. A. Costi, and D. Vollhardt, Phys. Rev. B 64, 045103 (2001), and references therein. 